Pacific Journal of Mathematic 


\title{
MEASURE ALGEBRAS ON IDEMPOTENT SEMIGROUPS
}

\author{
STEPHEN E. NEWMAN
}

Taylor has shown that for every commutative convolution measure algebra $M$ there is a compact topological semigroup $S$, called the structure semigroup of $M$, and an embedding $\mu \rightarrow \mu_{S}$ of $M$ into $M(S)$ such that every complex homomorphism of $M$ has the form $h_{f}(\mu)=\int_{S} f d \mu_{s}$ for some semicharacter $f$ on $S$.

This paper deals with commutative convolution measure algebras whose structure semigroups are idempotent. The measure algebra on the interval $[0,1]$, where the interval is given the semigroup operation of maximum multiplication, is an algebra of this type. These algebras are studied in this general setting in the hope of shedding new light on the known theory of measure algebras on locally compact idempotent semigroups and in the hope of extending attempts to classify a convolution measure algebra in terms of the algebraic nature of its structure semigroup.

An example is given of a measure algebra on a compact idempotent semigroup whose structure semigroup is not idempotent.

Our goal in this paper is to apply the structure theory for commutative convolution measure algebras developed by Taylor [5] to a special class of algebras which includes those studied by Hewitt and Zuckerman [3], Ross [4], and Baartz [1]. We will assume that each convolution measure algebra mentioned in this paper is commutative and, in addition, that each semigroup mentioned is commutative. We begin by giving the essential features of Taylor's structure theory.

A convolution measure algebra is roughly an ordered Banach space of measures with a multiplication which makes it a Banach algebra and which relates appropriately to the norm and the order. For a precise definition, see [5]. Examples include $L^{1}(G)$, the algebra of all absolutely continuous measures on a locally compact group $G ; M(G)$, the measure algebra on $G$ (all bounded regular Borel measures on $G$ ); and $M(S)$, the measure algebra on a locally compact semigroup $S$. Convolution is the multiplication operation in each of these examples. Both $L^{1}(G)$ and $M(G)$ are semisimple algebras as is $M(S)$ under certain not-too-restrictive conditions. We will therefore focus our attention only upon semisimple algebras.

Let $M$ denote a semisimple convolution measure algebra. Taylor has shown in [5] that there is a compact topological semigroup $S$, called the structure semigroup of $M$, and an embedding $\mu \rightarrow \mu_{S}$ of $M$ 
into $M(S)$ with the following properties.

(A) $\mu \rightarrow \mu_{S}$ is an algebraic isomorphism and an order preserving isometry.

(B) The image $M_{S}$ of the map $\mu \rightarrow \mu_{S}$ is weak* dense in $M(S)$; i.e., $M_{S}$ separates points in $C(S)$.

(C) $C(S)$ is the closed linear span of $\hat{S}$; i.e., $\widehat{S}$ separates points of $S$. ( $\hat{S}$ is the collection of all continuous semicharacters on $S$ ),

(D) Each complex homomorphism of $M$ has the form $h_{f}(\mu)=$ $\int_{S} f d \mu_{s}$ for some $f$ in $\hat{S}$.

The reader will recall that a semicharacter is a nonzero, bounded, complex valued function $f$ defined on the semigroup $S$ which satisfies $f(x \cdot y)=f(x) f(y)$ for all $x$ and $y$ in $S$. As a result of $(D)$, the set $\widehat{S}$ of semicharacters with the weak* topology induced by $M$ can be considered the maximal ideal space of $M$. We will regard a semicharacter $f$ in $S$ as both a continuous function on $S$ and a complex homomorphism of $M$ via the indentification given by (D), Thus, we write $f(\mu)$ in place of $h_{f}(\mu)$.

We are now in a position to define the type of algebra which will be our object of study.

Definition 1. A semisimple convolution measure algebra $M$ will be called a $P$-algebra provided $f(\mu) \geqq 0$ for every positive measure $\mu$ in $M$ and every complex homomorphism $f$ of $M$.

Examples of $P$-algebras are the measure algebra $M(T)$, under convolution, of the compact semigroup $T=[a, b]$ with multiplication $x \cdot y=\max \{x, y\}$ [3], and more generally, the measure algebra $M(T)$ of a finite product $T$ of locally compact, totally ordered spaces with co-ordinatewise maximum multiplication [1]. In both examples, each complex homomorphism of $M(T)$ has the form

$$
h_{A}(\mu)=\int_{T} \chi_{A} d \mu \quad \mu \in M(T),
$$

for some subsemigroup $A$ of $T$ whose complement $T \backslash A$ is a (prime) ideal of $T$ (Definition 1.5, [1]). Consequently, $M(T)$ is a $P$-algebra. In $\S 4$ we will give an alternate proof the $M(T)$ is a $P$-algebra, based on the results of $\S 3$.

We pause to define several terms with which the reader may not be familiar. The reader is referred to Taylor [5] for terms not defined here.

Let $M$ be a convolution measure algebra.

Definition 2. A closed subspace (subalgebra, ideal) $N$ of $M$ is 
called an $L$-subspace (subalgebra, ideal) if whenever $\mu \in N$, then $\nu \in N$ for all $\nu<\mu(\nu$ absolutely continuous with respect to $\mu)$.

Definition 3. An $L$-ideal $N$ of $M$ is called a prime $L$-ideal if $N^{\perp}=\{\mu \in \mu \mid \mu \perp \nu$ ( $\mu$ and $\nu$ are mutually singular) for all $\nu \in N\}$ is a subalgebra of $M$.

2. Some characterizations of $P$-algebras. Our first theorem gives six equivalent conditions for a semisimple convolution measure algebra $M$ to be a $P$-algebra. The identity $e$ mentioned in statements (5) and (6) of the theorem is the identity in $M$ if $M$ has an identity and is the identity adjoined to $M$ in the usual manner if $M$ does not have an identity. Similarly, the inversion mentioned in statement (6) takes place in the algebra $M$ if $M$ has an identity, and in the algebra " $M$ with identity adjoined" if $M$ does not have an identity.

THEOREM 1. Let $M$ be a semisimple convolution measure algebra. Then the following statements are equivalent.

(1) $M$ is a P-algebra.

(2) $\hat{S}$ is an idempotent semigroup.

(3) $S$ is an idempotent semigroup.

(4) For each $f \in \hat{S}, M=N_{f}+N_{f}^{\perp}$ where $N_{f}$ is a prime L-ideal such that if $\mu=\mu_{1}+\mu_{2}\left(\mu_{1} \in N_{f}, \mu_{2} \in N_{f}^{1}\right)$, then $f(\mu)=\left(\mu_{2}\right)_{S}(S)$.

(5) The spectral radius of $\mu-e$ is less than or equal to one for every positive measure $\mu$ of norm one.

(6) $\mu+e$ is invertible for every positive measure $\mu$ of norm one.

Proof. The order of proof will be $(1) \Rightarrow(2) \Leftrightarrow(3) \Rightarrow(4) \Rightarrow(1)$ and $(1) \Rightarrow(5) \Rightarrow(6) \Rightarrow(1) . \quad(1) \Rightarrow(2)$. Since the integral of each semicharacter in $\hat{S}$ with respect to any positive measure in $M$ is nonnegative, each semicharacter in $S$ is a nonnegative function by (B). Let $f$ be in $\hat{S}$. Then $f$ is nonnegative and hence for fixed $z, f^{z}$ is in $\widehat{S}$ if $\operatorname{Re} z>0$. Now $g_{s}(z)=f^{z}(s)$ is analytic in $\operatorname{Re} z>0$ for fixed $s \in S$. But then $g_{s}(z)$ is a nonnegative analytic function and is therefore constant. If we evaluate $g_{s}(z)$ at $z=1$, we obtain $f^{2}(s)=f(s)$ for all $\operatorname{Re} z>0$. If we let $z=2$ in the above equality, we obtain $f^{2}(s)=f(s)$ for each $s \in S$ and hence $f^{2}=f$. Therefore $\widehat{S}$ is an idempotent semigroup.

$(2) \Leftrightarrow(3)$. Let $f \in \widehat{S}$ and $s \in S$. We conclude that $f(s)=f^{2}(s)=$ $f(s) f(s)=f(s \cdot s)$ since $f^{2}=f$. Thus $s \cdot s=s$ by $(C)$, and hence $S$ is idempotent. Obviously, $(3) \Rightarrow(2)$.

$(3) \Rightarrow(4)$. If $S$ is idempotent, so is $\hat{S}$. Given $f \in \hat{S}$, let $J=$ $\{s \in S \mid f(s)=0\}$, We note that $J$ is a prime ideal in $S$. If we let $N_{f}=\left\{\mu \in M \mid \mu_{S}\right.$ is concentrated on $\left.J\right\}$, then $N_{f}$ is a prime $L$-ideal 
(Theorem 3.2, [5]) with orthogonal complement $N_{f}^{\perp}$. Thus if $\mu=\mu_{1}+$ $\mu_{2}$ where $\mu_{1} \in N_{f}$ and $\mu_{2} \in N_{f}^{\perp}$, then

$$
\begin{aligned}
& f(\mu)=\int_{s} f d \mu_{s}=\int_{s} f d\left(\mu_{1}+\mu_{2}\right)_{s}=\int_{J} f d\left(\mu_{1}\right)_{s}+\int_{s} f d\left(\mu_{2}\right)_{s}=\left(\mu_{2}\right)_{s}(S) . \\
& (4) \Rightarrow(1) . \quad \text { Obvious. } \\
& (1) \Rightarrow(5) . \quad \text { Recall that the spectral radius of an element } \chi \text { is a }
\end{aligned}
$$
Banach algebra (written $\|\chi\|_{s p}$ ) is given by $\|\chi\|_{s p}=\lim _{n \rightarrow \infty}\left\|\chi^{n}\right\|^{1 / n}$. If the algebra is commutative, then the spectral radius of $\chi$ is also the supremum norm of the Gelfand transform of $\chi$. If (1) holds, then, by definition, each complex homomorphism takes positive measures to nonnegative numbers. Let $\mu$ be a positive measure in $M$ of norm one. Then $0 \leqq f(\mu)=\widehat{\mu}(f) \leqq 1$ for every $f \in \hat{S}$. Therefore,

$$
1 \geqq \sup _{f \in \hat{S}}\left|(\mu-e)^{\wedge}(f)\right|=\left\|(\mu-e)^{\wedge}\right\|_{\infty}=\|\mu-e\|_{s p} .
$$

$(5) \Rightarrow(6)$. Let $\mu$ be a positive measure in $M$. Then $\mu /\|\mu\|$ is a positive measure of norm one and hence

$$
\left\|(\mu /\|\mu\|-e)^{\wedge}\right\|_{\infty}=\|\mu /\| \mu\|-e\|_{s p} \leqq 1 .
$$

Clearly $\hat{\mu}$ can never assume the value -1 . Thus $-1 \notin \sigma(\mu)$, and hence $\mu+e$ is invertible.

$(6) \Rightarrow(1)$. Let $\mu$ be a positive measure in $M$. If $\lambda>0$, then $\mu / \lambda+e$ is invertible. Hence $\mu+\lambda e$ is invertible and $-\lambda \notin \sigma(\mu)$. Therefore, the spectrum of any positive measure in $M$ contains no negative members. We claim that this fact ensures us that every positive measure will have real, nonnegative spectrum. For suppose $\mu$ is a positive measure whose spectrum is not real. Then there is an $f \in \hat{S}$ such that $\hat{\mu}(f)=\lambda=\lambda_{1}+i \lambda_{2}$ where $\lambda_{1}$ and $\lambda_{2}$ are real and $\lambda_{2} \neq 0$. We can choose a number $t>0$ such that $\exp (t \lambda)<0$. Thus $\exp (t \mu)$ is a positive measure with a negative number in its spectrum, a contradiction. The proof of the theorem is complete.

An $L$-subalgebra of a convolution measure algebra is again a convolution measure algebra. Since the spectral radius of a measure depends only upon the norms of the measure and its powers, statement (5) together with the above observation yields the following corollary.

Corollary. Every L-subalgebra of a P-algebra is a P-algebra.

3. A sufficient condition. Our next theorem gives a sufficient condition for an algebra to be a $P$-algebra. We suspect that the condition is also necessary but have not been able to prove it.

THEOREM 2. Let $M$ be a semisimple convolution measure algebra 
with structure semigroup $S$. If for every positive (nonzero) measure $\mu$ in $M$ there exist sequences $\left\{\mu_{n}\right\},\left\{\nu_{n}\right\}$ of positive measures in $M$ such that

(1) $\mu_{n} \rightarrow \mu$,

(2) $\mu_{n} * \nu^{\prime}=\mu_{n}(S) \nu^{\prime}$ for all $\nu^{\prime}<\nu_{n}$ and each $n$,

(3) $\nu_{n}<\sum_{m=0}^{\infty} \mu^{m} / 2^{m}$ for all $n$

then $M$ is a P-algebra.

Proof. Throughout this proof, $M$ is considered a subalgebra of $M(S)$. Let $\lambda$ be a positive measure in $M$, let $f$ be in $\hat{S}$, and let $J=$ $\{s \in S \mid f(s)=0\}$. Then $J$ is a prime ideal in $S$, and hence

$$
M_{J}=\{\nu \in M \mid \nu \text { is concentrated on } J\}
$$

is a prime $L$-ideal of $M$ with orthogonal complement $M_{J}^{\perp}$. Define $\mu$, $\mu^{\prime}$ by $\mu^{\prime}(E)=\lambda(E \cap J)$, for Borel sets $E$, and $\mu=\lambda-\mu^{\prime}$. Then $\mu \in M_{J}$ and $\mu^{\prime} \in M_{J}^{\perp}$.

If $\mu=0$, then $f(\lambda)=0$. If $\mu \neq 0$, then choose sequences $\left\{\mu_{n}\right\}$ and $\left\{\boldsymbol{\nu}_{n}\right\}$ guaranteed by the hypothesis of the theorem. We claim that there is a measure $\nu_{n}^{\prime}\left\langle\nu_{n}\right.$ such that $f\left(\nu_{n}^{\prime}\right) \neq 0$ for each $n$. If not, then for some $n, f\left(\nu_{n}^{\prime}\right)=\int f d \nu_{n}^{\prime}=0$ for all $\nu_{n}^{\prime}\left\langle\nu_{n}\right.$ and $f=0$ a.e. [ $\nu_{n}$ ]. Thus there is a Borel set $E \subset S$ of $\nu_{n}$-measure zero such that $f=0$ on $E$. Hence $\nu_{n} \in M_{J}$. But since $\mu \in M_{J}^{\perp}$ and $M_{J}^{\perp}$ is an $L$-subalgebra, $\sum_{m=0}^{\infty} \mu^{m} / 2^{m}$ is in $M_{J}^{\perp}$. Since $\nu_{n}<\sum_{m=0}^{\infty} \mu^{m} / 2^{m}, \nu_{n}$ is in $M_{J}^{\perp}$. Therefore, $\nu_{n} \perp \nu_{n}$ and so $\nu_{n}=0$, a contradiction. This establishes our claim.

Choose measures $\nu_{n}^{\prime}\left\langle\nu_{n}\right.$ such that $f\left(\nu_{n}^{\prime}\right) \neq 0$. Note that since $\mu_{n} * \nu_{n}^{\prime}=\mu_{n}(S) \nu_{n}^{\prime}$ and $f\left(\nu_{n}^{\prime}\right) \neq 0$, then, $f\left(\mu_{n}\right)=\mu_{n}(S) \geqq 0$. But $\mu_{n} \rightarrow \mu$; thus, $f\left(\mu_{n}\right) \rightarrow f(\mu)$. Therefore, $f(\mu)=\mu(S) \geqq 0$ and $f(\lambda)=f\left(\mu+\mu^{\prime}\right)=$ $f(\mu)+f\left(\mu^{\prime}\right)=f(\mu) \geqq 0$. Hence $M$ is a $P$-algebra.

4. An application of Theorem 2. If $T$ is an idempotent semigroup we can introduce a partial ordering " $\leqq$ " in $T$ by defining $x \leqq y$ if and only if $x \cdot y=y$ for all $x$ any $y$ in $T$. A totally ordered idempotent semigroup is one in which the above partial ordering is a total ordering. Our goal in this section is to show that the measure algebra on a finite product of totally ordered, locally compact, idempotent semigroups is a $P$-algebra. This result follows trivially from a theorem of Baartz (Theorem 3.5, [1]); however, we shall give an independent development using Theorem 2. We will need the three lemmas that follow.

LEMMA 1. Let $T$ be a locally compact idempotent semigroup and let $\mu$ and $\nu$ be in $M(T)$. Suppose $\operatorname{supp} \mu \leqq \operatorname{supp} \nu$ in the sense that 
for any $s \in \operatorname{supp} \mu$ and $t \in \operatorname{supp} \nu, s \leqq t(s \cdot t=t)$. Then $\mu * \nu=\mu(T) \nu$.

Proof. Supp $\mu$ denotes the support of the measure $\mu$. Let $A=$ $\operatorname{supp} \mu, B=\operatorname{supp} \nu$, and $E$ be a Borel subset of $T$. Then

$$
\begin{aligned}
\mu * \nu(E)= & \iint \chi_{E}(x \cdot y) d \mu(x) d \nu(y)= \\
& \int \mu\left(E_{y}\right) d \nu(y) \text { where } E_{y}=\{x \in T \mid x \cdot y \in E\} .
\end{aligned}
$$

But $\int_{T} \mu\left(E_{y}\right) d \nu(y)=\int_{B} \mu\left(E_{y}\right) d \nu(y)$ since $\nu$ is concentrated on $B$. Furthermore,

$$
\mu\left(E_{y}\right)= \begin{cases}0 & y \in B \backslash E \\ \mu(T) & y \in B \cap E\end{cases}
$$

since for $y \in B \cap E, A \subset E_{y}$ and for $y \in B \backslash E, A \cap E_{y}=\dot{\phi}$. Thus

$$
\int_{B} \mu\left(E_{y}\right) d \nu(y)=\mu(T) \nu(E)
$$

and hence $\mu * \nu=\mu(T) \nu$.

LEMma 2. Let $T$ be a totally ordered, locally compact, idempotent semigroup and let $\mu$ be a positive measure in $M(T)$. Then given $\varepsilon>0$, there is an $x \in T$ such that $\mu(\{y \in T \mid y \geqq x\})>0$ and $\mu(\{y \in T \mid y>x\})<\varepsilon$.

Proof. Since $\mu$ is a bounded regular measure, there is a compact set $K \subset \operatorname{supp} \mu$ such that $\mu(T \backslash K)<\varepsilon$. Let $z=\sup \{y \mid y \in K\}$. If $\mu(\{y \mid y \geqq z\})>0$, then the choice of $x=z$ completes the proof. If $\mu(\{y \mid y \geqq z\})=0$, we again apply the regularity of $\mu$ to obtain an $x<z$ such that $\mu(\{y \mid y>x\})<\varepsilon$. The choice of $z$ forces $\mu(\{y \mid y \geqq x\})>0$. The proof of the lemma is complete.

LEMma 3. Let $T=P_{i=1}^{m} T_{i}$ be a finite product of totally ordered, locally compact, idempotent semigroups $T_{i}$. Let $\mu$ be a positive measure in $M(T)$. Then given $\varepsilon>0$, there is an $x=\left(x_{1}, \cdots x_{m}\right)$ in $T$ such that $\mu^{m}(\{y \in T \mid y \geqq x\})>0$ and $\mu(T \backslash\{y \in T \mid y \leqq x\})<\varepsilon$.

Proof. Let $\pi_{i}$ be the projection map of $T$ onto $T_{i}$ for

$$
i=1,2, \cdots m \text {. }
$$

The measure $\mu_{i}=\mu_{\circ} \pi_{i}^{-1}$ is a positive measure in $M\left(T_{i}\right)$, Therefore, by Lemma 2 , there is an $x_{i}$ in $T_{i}$ such that $\mu_{i}\left(\left\{y \in T_{i} \mid y>x_{i}\right\}\right)<\varepsilon / m$ and $\mu_{i}\left(\left\{y \in T_{i} \mid y \geqq x_{i}\right\}\right)>0$. For notational convenience, let 


$$
J_{i}=\left\{y \in T_{i} \mid y>x_{i}\right\} \text { and } K_{i}=\left\{y \in T_{i} \mid y \geqq x_{i}\right\} .
$$

Then the above statement becomes $\mu_{i}\left(J_{i}\right)<\varepsilon / m$ and $\mu_{i}\left(K_{i}\right)>0$.

Let $x=\left(x_{1}, x_{2}, \cdots x_{m}\right)$. We first note that

$$
T \backslash\left\{y \in T \mid y \leqq x=\bigcup_{i=1}^{m} \pi_{i}^{-1}\left(J_{i}\right) .\right.
$$

Thus $\mu(T \backslash\{y \in T \mid y<x\})=\mu\left(\bigcup_{i=1}^{m} \pi_{i}^{-1}\left(J_{i}\right)\right) \leqq \sum_{i=1}^{m} \mu \circ \pi_{i}^{-1}\left(J_{i}\right)<\sum_{i=1}^{m} \varepsilon / m=\varepsilon$. We next note that

$$
\pi_{1}^{-1}\left(K_{1}\right) \cdot \pi_{2}^{-1}\left(K_{2}\right) \cdots \pi_{m}^{-1}\left(K_{m}\right)=\bigcap_{i=1}^{m} \pi_{i}^{-1}\left(K_{i}\right)=\{y \in T \mid y \geqq x\} .
$$

Since $\mu$ has mass on each of the sets $\pi_{i}^{-1}\left(K_{i}\right), \mu^{m}$ has mass on

$$
\pi_{1}^{-1}\left(K_{1}\right) \cdot \pi_{2}^{-1}\left(K_{2}\right) \cdots \pi_{m}^{-1}\left(K_{m}\right) ; \text { i.e., } \mu^{m}(\{y \in T \mid y \geqq x\})>0 .
$$

This establishes the lemma.

THEOREM 3. Let $T=P_{i=1}^{m} T_{i}$ be a finite product of totally ordered, locally compact, idempotent semigroups $T_{i}$. Then the measure algebra $M(T)$ is a P-algebra.

Proof. Let $\mu$ be a positive measure in $M(T)$. Lemma 3 guarantees the existence of a sequence $\left\{x_{n}\right\}_{n=1}^{\infty}$ in $T$ such that

$$
\mu\left(T \backslash\left\{y \in T \mid y \leqq x_{n}\right\}<1 / n\right.
$$

and $\mu^{m}\left(\left\{y \in T \mid y \geqq x_{n}\right\}>0\right.$.

Let $\mu_{n}=\mu \mid\left\{y \in T \mid y \leqq x_{n}\right\}$ and let $\nu_{n}=\mu^{m} \mid\left\{y \in T \mid y \geqq x_{n}\right\}$. Here we denote the measure $\mu$ restricted to a set $A$ by $\mu \mid A(\mu \mid A(E)=$ $\mu(A \cap E)$ for any Borel set $E)$. We claim that the sequences $\left\{\mu_{n}\right\}$ and $\left\{\nu_{n}\right\}$ satisfy the hypothesis of Theorem 2 . Clearly, $\mu_{n} \rightarrow \mu$. Since $\operatorname{supp} \mu_{n} \leqq \operatorname{supp} \nu_{n}$, and since $\operatorname{supp} \nu_{n}^{\prime} \subset \operatorname{supp} \nu_{n}$ for any $\nu_{n}^{\prime}<\nu_{n}$, Lemma 1 assures us that $\mu_{n} * \nu_{n}^{\prime}=\mu_{n}(T) \nu_{n}^{\prime}$. Finally, $\nu_{n}$ is a nonzero measure such that $\nu_{n}<\mu^{m}<\sum_{k=0}^{\infty} \mu^{k} / 2^{k}$. Since $M(T)$ is semisimple [1], $M(T)$ is a $P$-algebra by Theorem 2 .

5. A counterexample. Theorem 1 shows that any $P$-algebra may be considered as an $L$-subalgebra of the measure algebra on a compact idempotent semigroup. Each of the examples given in $\S 1$ is a measure algebra on a locally compact idempotent semigroup (not the structure semigroup). It is therefore natural to ask whether or not the measure algebra on any locally compact, idempotent semigroup is a $P$-algebra. The answer to this question is "no" as the counterexample of this section will show. We first make the following definition. 
Definition 4. A subset $Q$ of an idempotent semigroup $S$ will be called independent if whenever $x_{1} \cdot x_{2} \cdots x_{n}=y_{1} \cdot y_{2} \cdots y_{m}$ for $\left\{x_{i}\right\}_{i=1}^{n} U$ $\left\{y_{j}\right\}_{j=1}^{m} \subset Q$ and $m<n$, then $x_{i}=y_{j}$ for some $i$ and $j(1 \leqq i \leqq n$ and $1 \leqq j \leqq m)$.

Let $C$ denote the Cantor set on the interval $[0,1]$. Let $S$ denote the collection of all finite subsets of $C$ and let union be the semigroup operation in $S$. Note that the one point sets form an independent subset of $S$ in the sense of Definition 4. For an open-compact subset $U \subset C$, $X \in S$, define

$$
\chi_{U}(X)=\left\{\begin{array}{lll}
1 & \text { if } & X \subset U \\
0 & \text { if } & X \not \subset U
\end{array} .\right.
$$

Give $S$ the weak topology generated by the functions $\left\{\chi_{U}\right\}$ ( $U$ opencompact). Observe that each $\chi_{U}$ is a continuous semicharacter on $S$. Let $V$ be a countable open-compact base for $C$ and let $\widetilde{V}=\{\widetilde{U} \mid U \in V\}$. Finally, let $\left\{U_{i}\right\}_{i=1}^{\infty}=V \cup \widetilde{V}$ and note that the family $\left\{\chi_{U_{i}}\right\}_{i=1}^{\infty}$ separates points in $S$.

Let $T$ be the countable topological product of the two-point semigroup $\{0,1\}$, under multiplication. Thus $T$ is a compact idempotent semigroup. We now define a map $\alpha: S \rightarrow T$ by $[\alpha(X)]_{i}=\chi_{U_{i}}(X)$ for any $X \in S$. Note that $\alpha$ is a continuous one-to-one homomorphism from $S$ into $T$. We further observe that $C$ is embedded in an obvious way in $S$, and hence in $T$, as an independent set.

The concluding argument is similar to the one given in the HewittKakutani paper on $M(G)$ [2]. There is a positive continuous measure $\mu$ of norm one concentrated on $C$. Using Fubini's theorem and the fact that $C$ is independent, it can be shown that $\mu$ and all its powers are mutually singular [2]. Now let $\sigma=\delta_{e}-\mu(e$ is the identity in $T)$. Then $\left\|\sigma^{n}\right\|=\left\|\sum_{k=0}^{n} C_{n, k}(-1)^{k} \mu^{k}\right\|=\sum_{k=0}^{n} C_{n, k}=2^{n}$. Hence

$$
\|\hat{\sigma}\|_{\infty}=\lim _{n \rightarrow \infty}\left\|\sigma^{n}\right\|^{1 / n}=2 \text {. }
$$

Thus there is a complex homomorphism $h$ of $M(T)$ such that $|h(\sigma)|=2$. This forces $h(\mu)=-1$. Therefore, $M(T)$ is not a $P$-algebra.

The countable product of the two point semigroup, with the operation of coordinatewise multiplication, is a sub-semigroup of the countable product of unit intervals, with the operation of coordinatewise minimum. Thus although the measure algebra on a finite product of intervals with coordinatewise maximum multiplication is a $P$-algebra, this is not the case for an infinite product of intervals. We are therefore led to conjecture that a measure algebra $M(T)$ is a $P$-algebra if and only if $T$ is an idempotent semigroup which satisfies a certain "finite dimensionality" condition. 


\section{BIBLIOGRAPHY}

1. A. P. Baartz, The measure algebra of a locally compact semigroup, Pacific J. Math. 21 (1967), 199-213.

2. E. Hewitt and S. Kakutani, A class of multiplicative linear functionals on the measure algebra of a locally compact abelian group, Illinois J. Math. 4 (1960), 553-574. 3. E. Hewitt and H. S. Zuckerman, Structure theory for a class of convolution algebras, Pacific J. Math. 7 (1957), 913-941.

4. K. A. Ross, The structure of certain measure algebras, Pacific J. Math. 11 (1961), $723-736$.

5. J. L. Taylor, The structure of convolution measure algebras, Trans. Amer. Math. Soc. 119 (1965), 150-166.

Received May 30, 1968.

UNIVERSITY OF UTAH

Salt Lake City, Utah

AND

UNIVERSITY OF MISSOURI-ST. LOUIS 



\section{PACIFIC JOURNAL OF MATHEMATICS}

\section{EDITORS}

H. ROYDEN

Stanford University

Stanford, California

Richard Pierce

University of Washington

Seattle, Washington 98105
J. DUGUNDJI

Department of Mathematics

University of Southern California

Los Angeles, California 90007

BASIL GORDON

University of California

Los Angeles, California 90024

\section{ASSOCIATE EDITORS}
E. F. BECKENBACH
B. H. NEUMANN
F. WOLF
K. YosHIDA

\section{SUPPORTING INSTITUTIONS}

UNIVERSITY OF BRITISH COLUMBIA

CALIFORNIA INSTITUTE OF TECHNOLOGY

UNIVERSITY OF CALIFORNIA

MONTANA STATE UNIVERSITY

UNIVERSITY OF NEVADA

NEW MEXICO STATE UNIVERSITY

OREGON STATE UNIVERSITY

UNIVERSITY OF OREGON

OSAKA UNIVERSITY

UNIVERSITY OF SOUTHERN CALIFORNIA
STANFORD UNIVERSITY

UNIVERSITY OF TOKYO

UNIVERSITY OF UTAH

WASHINGTON STATE UNIVERSITY

UNIVERSITY OF WASHINGTON

*

AMERICAN MATHEMATICAL SOCIETY CHEVRON RESEARCH CORPORATION TRW SYSTEMS

NAVAL WEAPONS CENTER 


\section{Pacific Journal of Mathematics}

\section{Vol. 31, No. $1 \quad$ November, 1969}

James Burton Ax, Injective endomorphisms of varieties and schemes........

Richard Hindman Bouldin, A generalization of the Weinstein-Aronszajn

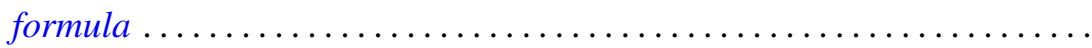

John Martin Chadam, The asymptotic behavior of the Klein-Gordon equation with external potential. II ...............................

Rina Hadass, On the zeros of the solutions of the differential equation

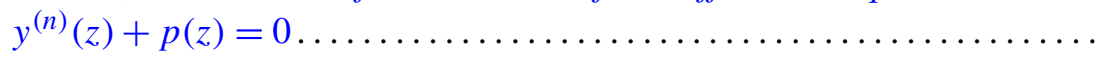

John Sollion Hsia, Integral equivalence of vectors over local modular lattices. II .............................................

Robert Hughes, Boundary behavior of random valued heat polynomial

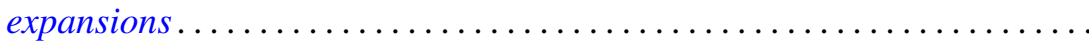

Surender Kumar Jain, Saad H. Mohamed and Surjeet Singh, Rings in which every right ideal is quasi-injective .........................

T. Kawata, On the inversion formula for the characteristic function .........

Erwin Kleinfeld, On right alternative rings without proper right ideals......

Robert Leroy Kruse and David Thomas Price, On the subring structure of

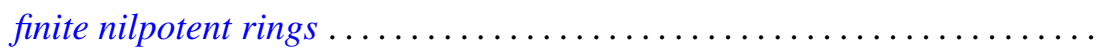

Marvin David Marcus and Stephen J. Pierce, Symmetric positive definite multilinear functionals with a given automorphism. .

William Schumacher Massey, Pontryagin squares in the Thom space of a bundle...

William Schumacher Massey, Proof of a conjecture of Whitney ...

John William Neuberger, Existence of a spectrum for nonlinear transformations

Stephen E. Newman, Measure algebras on idempotent semigroups ...

$\mathrm{K}$. Chandrasekhara Rao, Matrix transformations of some sequence spaces

Robert Bruce Schneider, Some theorems in Fourier analysis on symmetric

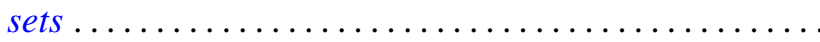

Ulrich F. K. Schoenwaelder, Centralizers of abelian, normal subgroups of hypercyclic groups...

Jerrold Norman Siegel, $G$-spaces, $H$-spaces and $W$-spaces

Robert Irving Soare, Cohesive sets and recursively enumerable Dedekind cuts...

Kwok-Wai Tam, Isometries of certain function spaces .... . .

Awadhesh Kumar Tiwary, Injective hulls of semi-simple modules over regular rings ....

Eldon Jon Vought, Concerning continua not separated by any nonaposyndetic subcontinuum .................... 